\title{
Un concept évolutif, une actualité en débat
}

L'éducation artistique en Australie

Art education in Australia

La educación artística en Australia

\section{Anne Bamford}

Traducteur : Yves Goujon et Daniel Zafrani

\section{OpenEdition}

\section{Journals}

Édition électronique

URL : https://journals.openedition.org/ries/1089

DOI : 10.4000/ries. 1089

ISSN : 2261-4265

Éditeur

France Education international

Édition imprimée

Date de publication : 1 septembre 2006

Pagination : 55-67

ISSN : 1254-4590

Référence électronique

Anne Bamford, "Un concept évolutif, une actualité en débat », Revue internationale d'éducation de Sèvres [En ligne], 42 I septembre 2006, mis en ligne le 10 novembre 2011, consulté le 08 juillet 2021. URL : http://journals.openedition.org/ries/1089; DOI : https://doi.org/10.4000/ries.1089 


\section{Un concept évolutif, une actualité en débat}

\section{L'éducation artistique en Australie*}

\section{Anne Bamford}

Cet article présente une vue d'ensemble de l'éducation artistique en Australie et souligne les principaux contextes historiques ayant des effets sur l'enseignement de l'art et de la culture dans ce pays. Il s'agit d'une synthèse de plusieurs années de recherche et de réflexion sur le contexte australien et plus récemment, d'une comparaison entre l'Australie et les standards internationaux.

\section{LA DÉFINITION DES ARTS DANS LE SYSTÈME ÉDUCATIF}

L'expression «enseignement artistique» en Australie est généralement utilisée pour parler de l'enseignement des arts visuels. Il s'agit d'une formule globale pour décrire les arts visuels, les objets réalisés à la main et l'enseignement de l'esthétique. D’un point de vue général, l'art à l'école primaire tend à être plus manuel alors que dans le secondaire, on vise un rééquilibrage entre théorie et pratique. De la même façon, à l'école primaire, les enseignants sont censés initier les élèves aux disciplines artistiques et utiliser ces enseignements dans le cadre d'autres disciplines du curriculum, alors que dans le secondaire, les enseignants mettent l'accent sur l'enseignement des disciplines artistiques, sans viser la réutilisation de ces disciplines dans d'autres enseignements.

Il existe en Australie un large éventail de pratiques d'enseignement des disciplines artistiques dans le cadre d'enseignements aussi bien formels qu'informels et ceci à tous les niveaux de la scolarité. Ce phénomène reflète bien le rôle significatif que jouent les artistes, la collectivité et tout un ensemble d'autorités légales et d'organisations non gouvernementales (parmi lesquelles les galeries) dans l'offre australienne en matière d'éducation artistique.

\section{L’éducation artistique dans le curriculum}

L'éducation artistique est obligatoire dans tous les États dans le cadre du curriculum. Ce dernier correspond à tout type de document produit à un niveau centralisé et définissant la nature et la portée de l'enseignement des matières artistiques et culturelles dans les écoles australiennes. Cela comprend le programme des cours, les enseignements dispensés, le matériel pédagogique,

\footnotetext{
* Article traduit par Daniel Zafrani et Yves Goujon.
} 
les communiqués, la politique à suivre et tout ce qui est produit, au niveau central, pour guider l'organisation et la mise en ouvre de l'éducation artistique et de l'offre culturelle dans les établissements scolaires.

Bien qu'il soit difficile d'obtenir des chiffres exacts - car il existe un écart entre ce qui est obligatoire, ce qui est choisi et ce que les enfants reçoivent effectivement -, les enfants âgés de cinq à quinze ans suivent en gros soixante à quatrevingts minutes par semaine de cours d'éducation artistique obligatoire. Ils sont également susceptibles de recevoir un enseignement artistique et culturel complémentaire par le biais de visites, de représentations théâtrales, de pédagogie intégrée, de choix et de préférences déterminés par les enfants ou par les enseignants. Entre treize ans et dix-huit ans, les enfants peuvent choisir des cours facultatifs dans toutes les disciplines artistiques et, dans la plupart des États, il existe un grand choix de cours optionnels dans le domaine artistique, bien que ce qui est réellement proposé dans les écoles varie d'un établissement à l'autre - plus particulièrement dans les petits établissements régionaux ou ruraux.

Cette éducation artistique officielle est complétée par un éventail de programmes offrant des représentations théâtrales et des expositions à l'école, des sorties culturelles et artistiques, des découvertes d'artistes, etc. La qualité de l'offre non officielle disponible dans les écoles, les collèges et les lycées est en général bonne et les institutions culturelles accordent une grande attention à l'éducation et aux jeunes dans leurs programmes.

Alors que les exigences, en matière d'arts et de culture, sont inscrites dans la loi et précisées dans le curriculum, les pratiques en classe portent le nom de «programme artistique et culturel». Ces «programmes» sont les ressources et les dispositifs mis en place au niveau de l'établissement pour que soit mis en œuvre le curriculum. Cela peut comprendre les projets développés par l'enseignant, les projets éducatifs dans des musées, des collaborations avec des artistes et avec la communauté, l'évaluation, les ressources documentaires diverses (manuels, documentation en ligne, estampes, etc.), l'emploi du temps, des critères de sélection établis par l'école, etc. Cette liste n'est bien entendu pas exhaustive.

Ces programmes sont mis en place par les enseignants. J'entends par «enseignant» tout professionnel directement en charge de l'offre d'éducation artistique dans un programme donné, à tous les niveaux et dans toute la variété des contextes scolaires couverts par le curriculum. Cette définition large tient compte de la tendance croissante, en Australie, à impliquer les professionnels du milieu de l'art et les organismes culturels dans l'éducation artistique.

\section{DÉBATS ET INTERROGATIONS SUR L'ENSEIGNEMENT ARTISTIQUE}

Les études australiennes sur l'éducation artistique (Ashton 1999) présentent de nombreuses similitudes avec les études internationales qui montrent que, généralement, la qualité est variable et, dans plusieurs cas, au-dessous 
des standards susceptibles de garantir aux enfants une éducation artistique de qualité (Bamford 2006).

Les raisons communément avancées pour expliquer ce défaut de qualité tiennent au fait que les arts sont perçus comme facteurs de perturbation dans la classe. Par conséquent, de nombreux enseignants recherchent des activités qui permettent un contrôle strict du comportement des enfants et utilisent des ressources limitées et «faciles à nettoyer». Comme l'indique Ashton (1998:8), «dans nos écoles primaires, l'art sert souvent de vitrine, (à un rythme) spasmodique, et son statut dans le curriculum ressemble à une zone floue comprenant des documents systématiques peu nombreux et mal répartis ».

Alors que l'enseignement des arts et de la culture tend à être d'un niveau plus élevé dans le secondaire supérieur - où les enseignants reçoivent généralement une formation spécialisée -, les professeurs des écoles adoptent fréquemment des approches centrées sur l'enfant, optant pour des tâches individuelles sans limites de durée dans lesquelles l'enseignant se pare d'un rôle positif et d'encouragement mais ne fournit presque pas d'instruction spécifique.

Ces dernières années, l'influence de la technologie (Education 1989; Board od Studies 2000) se fait sentir dans la totalité des objectifs assignés à l'éducation artistique. Il existe un accord tacite qui veut que l'enseignement artistique soit centré sur la technologie et soit ainsi plus fonctionnel, particulièrement dans le domaine des multimédias interactifs. Cette diversité dans les objectifs est vue par Speck comme un obstacle pour l'enseignement artistique. Elle écrit à ce propos que «cet inventaire d'objectifs par ailleurs louables conduirait à ce que l'enseignant, dans sa classe, perde de vue la structure de sa discipline et que, de ce fait, une discipline inscrite au curriculum et déjà affectée par l'ampleur d'un mythe le soit davantage encore par la diversité des objectifs ».

Fondés sur l'idée que tout ce qu'on demande aux enseignants est de fournir aux enfants un ensemble de matériaux et de produits à réaliser avec succès, les curricula en arts et en culture ont plutôt visé la diversité de ce qui pouvait être montré qu'un approfondissement de la compréhension et un développement régulier.

Alors que les difficultés de l'enseignement artistique débordent largement du cadre de la salle de cours, l'environnement dans lequel les enseignants doivent dispenser leurs enseignements sert davantage à gérer les difficultés rencontrées lors de la mise en œuvre d'un enseignement artistique de qualité. Les enseignants sont confrontés à un dilemme : comment concilier les exigences du curriculum avec les réalités du contexte comme le manque de temps, d'espace ou de documentation? Malgré des techniques de communication en développement et un accès croissant aux informations, l'enseignement artistique se heurte aux limites physiques de la salle de classe.

Récemment, le gouvernement australien s'est penché sur les effets et l'impact des industries culturelles dans l'économie australienne et sur le besoin 
urgent d'améliorer l'offre d'éducation artistique dans les écoles. Un certain nombre d'études ont été lancées pour aider collectivement à définir des objectifs et à fournir des données utilisables afin de faire correspondre les programmes et les besoins des élèves et des professeurs dans ce domaine, ainsi que ceux de la société dans son ensemble.

En Australie, l'engouement actuel pour l'enseignement artistique et culturel se fonde sur l'idée que cet enseignement offre aux jeunes l'opportunité d'apprentissages riches et complexes, ainsi que d'expériences marquantes, en encourageant les qualités personnelles, sociales, culturelles et cognitives. De plus, on considère que l'enseignement artistique et culturel développe des esprits créatifs et inventifs ayant les compétences nécessaires pour la société de la connaissance et qu'il fournit la possibilité d'établir des contacts et des expériences sociales intéressants.

Les transformations économiques, technologiques, politiques et dans le secteur des communications continuent d'avoir de profondes conséquences sur le contenu, la transmission et la pratique de l'enseignement artistique et culturel en Australie. La nature constamment évolutive de la pratique des arts contemporains s'ajoute à la difficulté de déterminer l'étendue et la qualité de l'éducation artistique à l'école.

Alors que le gouvernement australien reconnaît l'utilité de l'enseignement artistique, ce discours politique n'a pas été confirmé par des changements significatifs dans les pratiques. Ce point est particulièrement vérifiable dans la formation initiale des enseignants où l'enseignement artistique continue d'être le parent pauvre en termes d'ambition, de temps et de profondeur intellectuelle. Les professeurs stagiaires ne disposent pas de beaucoup de temps pour réfléchir ou pour s'engager dans un examen critique de leur discipline, dans la mesure où ils reçoivent seulement le strict minimum en termes de formation artistique requise préalable à leur entrée en fonction. En outre, les étudiants eux-mêmes considèrent qu'une grande partie de leur formation pédagogique est peu intéressante car trop abstraite et théorique. L'apprentissage est hors contexte et il est caractérisé par une forte concurrence pour l'obtention des meilleures notes plutôt que par la mise en place de stratégies d'apprentissage en groupe ${ }^{1}$. Selon Boughton $^{2}$, de nombreux «modèles de formations des enseignants sont fréquemment inappropriés aux écoles australiennes contemporaines ». Wright ${ }^{3}$ résume les problèmes inhérents à la formation des professeurs à trois grandes raisons : «le statut social peu élevé des professeurs d'écoles maternelles et primaires; le manque de temps et le fait que l'accent est insuffisamment mis sur les sujets artistiques au sein des établissements de formation des maîtres; une expérience artistique et des capacités plutôt faibles des professeurs en formation ».

1. Brady, Segal et al. 1998.

2. Boughton (1999:59).

3. Wright (1989:8). 
Pour Duncum ${ }^{4}$ comme pour Taylor, la formation initiale des professeurs des écoles généralistes est le trou noir de l'éducation artistique. "Parce que la société voit et évalue l'importance du degré d'alphabétisation et des capacités à compter, elle s'attend à ce que les futurs professeurs des écoles aient obtenu un minium de compétences dans ces domaines qui constituent l'essentiel du contenu des cours. Malheureusement, on n'accorde que très peu d'intérêt à ce que l'on pourrait considérer comme l'alphabétisation visuelle et qui constitue une part importante de nos vies ${ }^{5}$.

Ashton conclut au besoin sérieux de mettre au défi la sensibilité artistique des professeurs des écoles eux-mêmes. L'enseignement artistique actuellement dispensé en Australie tend à favoriser les pédagogues plutôt que ceux qui ont suivi des études d'art. On passe beaucoup de temps sur les théories de l'enseignement de l'art et sur les conséquences de ces connaissances en termes de curriculum (Ashton 1999).

\section{L'ENSEIGNEMENT AUSTRALIEN ET SES INFLUENCES HISTORIQUES}

L'Australie est un pays multiculturel composé de groupes ethniques divers, et depuis peu, les cultures aborigènes et asiatiques sont davantage présentes dans la conception et la mise en œuvre du programme artistique et culturel. Traditionnellement, les Aborigènes ne possèdent pas un mot unique pour désigner l'"art». Ils voient l'art comme si intimement lié à la spiritualité et à la culture qu'il est gravé dans ces deux concepts. Au sein des cultures traditionnelles et contemporaines, l'art et l'imaginaire jouent un rôle vital dans tous les aspects de la vie. Les Rêves aborigènes sont transmis à travers l'art et les gardiens du langage imagé et des figures emblématiques visuelles de cette culture sont au sommet de la hiérarchie sociale.

Depuis son invasion européenne et sa colonisation il y a plus de deux cents ans, l'Australie est un pays d'immigrés apportant avec eux, de tous les coins du monde, leurs compétences, leur savoir et leurs croyances. Ces dernières années, le développement de la mondialisation et des communications a fait disparaître la barrière de l'éloignement de l'Australie et, dans l'enseignement, l'accent est plutôt mis sur le monde que sur les réalités locales. Les liens se font à l'échelle de la planète, se complexifient, et théorie et pratique deviennent l'objet d'appropriations internationales (Freedman et Hernandez 1998 : 183 et 187).

Le résultat de cette internationalisation est qu'il semble qu'il existe des objectifs communs relativement établis qui sont généralement acceptés au niveau international et qui ont un effet direct sur les pratiques nationales. Selon Errazuriz (1998), il n'est pas toujours recommandé ou possible de justifier un

\footnotetext{
4. Duncum (1999: 15).

5. Taylor (1998: 256).
} 
enseignement artistique local par rapport à ces objectifs étrangers et diffusés à un niveau international.

Les facteurs historiques et culturels façonnent l'élaboration de l'éducation artistique en Australie et la manière dont les théories générales sur l'enseignement de l'art sont transmises et mises en place dans le contexte scolaire. Alors que les enseignants en arts peuvent se permettre d'être relativement ignorants des discours sur l'enseignement des arts qui façonnent leurs préférences pédagogiques, il est cependant utile de mettre en évidence les sources politiques et historiques qui modèlent encore les pratiques de l'enseignement de l'art dans les écoles australiennes.

Les sections suivantes proposent une organisation simplifiée de la diversité des influences qui pèsent sur l'enseignement de l'art et de la culture en Australie. Elles sont présentées dans l'ordre approximatif de leur apparition au sein de l'enseignement australien mais se chevauchent partiellement.

\section{Les arts appliqués à l'industrie}

Le cadre théorique technocratique de l'éducation artistique est apparu à la fin du XIX ${ }^{\mathrm{e}}$ siècle. L'art était alors synonyme de dessin et l'on valorisait la copie et la technique de la perspective. On dissuadait les étudiants d'être inventifs et expressifs et le principal objectif dans les écoles était de former «l'œil, la main, et l'esprit» (Freedman 1987 : 66). Pendant cette période, l'art devint part du secteur productif et les techniques étaient primordiales au savoir ou au développement conceptuels.

En Australie, l'influence du mouvement artistique industriel comprenait l'introduction des travaux manuels dans les écoles. La plus grande partie de leur enseignement était fondée sur les techniques et l'art était apprécié pour ses applications fonctionnelles dans l'architecture, la photographie et la conception du mobilier. L'art populaire et les travaux manuels gagnèrent en popularité, particulièrement dans les écoles primaires, car ils permettaient d'aider les enfants à développer leur coordination œil/main. On observe alors une distinction claire entre les travaux manuels et les beaux-arts. Pendant les cours de travaux manuels, on enseignait la menuiserie/ébénisterie aux garçons et la couture aux filles. Le conformisme était plus apprécié que l'individualisme et les enseignants suivaient des programmes séquencés et normalisés.

\section{Le dessin d'enfant ${ }^{6}$}

Selon Boughton (1998), jusqu’à la fin des années trente, on utilisait le terme «art» pour désigner le dessin dans les écoles primaires en Australie.

6. NdT: Child art prend ici le sens de «dessin d'enfant».

$\mathrm{NdT}$ : Child art désigne le dessin d'enfant, mais cette expression fait également référence à l'éducation artistique précoce. Elle englobe ici d'autres formes artistiques. L'expression est donc parfois traduite par «éducation artistique précoce». 
L'expression de la créativité personnelle était clairement élevée en dogme dans les objectifs prescrits par le «Programme pour les écoles primaires» (Wales 1952 : 357). Le premier « objectif de base de l'art à l'école primaire » était d'encourager les enfants à «exprimer librement leurs expériences, leurs élans créatifs et imaginatifs selon leurs propres critères, en prenant soin de permettre aux forces créatives de l'enfant de croître aussi librement que possible à chaque stade de son développement».

Le mouvement pour l'éducation artistique précoce est le fruit du travail de théoriciens du développement de l'enfant et de l'aspect psychologique de l'étude de l'art produit par les enfants. Selon ce mouvement, les pratiques artistiques des enfants étaient exploitables pour développer leur intelligence et leur ouverture d'esprit. Les professeurs australiens d'éducation artistique ont adopté un rôle de médiateurs, en proposant un environnement stimulant pour l'enfant, du matériel adapté et varié, des encouragements pour les efforts fournis, sans toutefois diriger ou influencer leur processus créatif. Selon Holt (1997), il s'agit d'une «action passive» car l'intervention de l'adulte était perçue comme nocive à l'expression artistique innée des enfants.

En Australie, le programme s'est développé en fonction des étapes du développement de l'enfant et a mis l'accent sur la manière dont les cours pouvaient s'organiser pour encourager ce développement. L'enseignant était supposé apprendre de l'enfant, puisque l'enfant était une source d'inspiration et d'énergie créative. Cela a généré une tension entre le rôle supposé de l'enseignant et les limites de ce que les dessins d'enfants et d'adultes ont en commun. Les professeurs sont devenus moins clairs quant à leur rôle dans l'éducation artistique et le programme scolaire australien fit référence à des concepts tels que «créativité», "sentiment» et «expression»sans pour autant clarifier leur portée et leur applicabilité.

\section{L'art en tant qu'expression}

Au mouvement en faveur de l'éducation artistique précoce ${ }^{7}$, il convient d'ajouter celui de l'art comme moyen d'expression, mouvement solidement fondé sur la croyance que l'art était vital au bien-être émotionnel de l'enfant. On considérait que l'art était un espace de liberté et de détente qui garantissait un enseignement équilibré, satisfaisant les besoins intellectuels et émotionnels de l'enfant. On pensait que l'art était une activité ludique qui contrebalançait la nature rigide des activités plus académiques du programme australien.

S'est ajoutée à la notion d'expression individuelle l'idée que l'art pourrait être utilisé pour guérir l'âme et mieux préparer l'enfant à affronter une société cruelle. On pensait également que l'expression thérapeutique à travers 
l'art répondait à des préoccupations sociales liées aux effets de la guerre, des pressions économiques, de la violence grandissante et de l'aliénation au sein de la société. Pour procurer un bienfait thérapeutique, le processus artistique créatif était plus important que la production elle-même. Des mentions dans les programmes ont commencé à souligner l'importance du "processus créatif». À partir de 1974, l'éducation artistique en Australie fut directement perçue comme "un processus» impliquant l'acquisition de connaissances, d'aptitudes, d'habitudes et de dispositions, ainsi que le développement d'une sensibilité esthétique. Le programme de 1989 (Education 1989), dont la totalité du contenu était organisée selon une suite de rubriques sur les processus et les pratiques, est allé encore plus loin. Les processus incluaient la perception, la réaction, l'évaluation, l'organisation et la manipulation (Education 1989 : 16-17). Les processus créatifs étaient appréciés pour les effets qu'ils procuraient sur les individus. La qualité de la pratique, plus que la qualité de la production de l'enfant, constituait l'objectif premier de l'éducation artistique.

\section{L'art en tant que connaissance}

Dans les années quatre-vingts, l'éducation artistique en Australie a commencé à glisser vers une organisation plus méthodique et plus intellectuelle. Les professeurs australiens d'éducation artistique se sont regroupés en corps professionnels puissants, désireux de promouvoir leur discipline. Pour réagir à une mise à l'écart visible de l'art dans le domaine des activités récréatives d'expression, les enseignants de disciplines artistiques se sont joints à leurs confrères d'autres pays (notamment les États-Unis d'Amérique et le Royaume-Uni) afin d'affirmer la rigueur intellectuelle et le fondement théorique qui sous-tendent l'éducation artistique.

On est passé d'un raisonnement qui se justifiait par les émotions et le comportement de l'enfant à des approches cognitives. Ajouté à cela, l'accent mis sur l'individualisation a été inclus dans un ensemble qui valorisait l'importance de la connaissance des œuvres culturelles et historiques représentant les meilleures pratiques artistiques (Greer 1992). L'approche cognitive de l'art s'est éloignée des modèles inspirés par les enfants, pour s'appuyer sur les études des œuvres d'art montrées comme exemples des meilleures pratiques dans ce domaine. Des termes comme «solide», «imaginatif» et «élans», issus du dessin d'enfant, ont été remplacés par "analytique», "évaluation», "connaissance de la forme», et " autocritique».

\section{L'art comme éducation esthétique et visuelle}

Au début du vingtième siècle, l'éducation artistique en Australie suivait de près la perception esthétique européenne. L'esthétique était associée à la «civilisation et à la culture». Selon les conceptions française et italienne, qui 
considèrent les disciplines artistiques comme une réalisation culturelle et un signe visible d'éducation et d'appartenance à une classe sociale, on avait le sentiment que l'étude de la musique, du théâtre classique, des beaux-arts et de l'architecture était une activité de l'esprit. L'étude de la dimension esthétique était vue comme une activité enrichissant l'intelligence et la culture de l'enfant. La préface de The Art reader, texte fondateur sur l'éducation artistique en Australie, (Quinn 1905 : 3) traite de l'importance du développement de la conscience esthétique dans le "pays neuf » qu'était l'Australie. La façon d'appréhender l'esthétique chez Quinn tient compte des styles classiques européens et déplore l'esthétique des villes australiennes.

Voir l'art comme une richesse esthétique est une idée qui réapparut de façon plus succincte en 1974 dans le programme K-6 ${ }^{8}$ des arts visuels (Wales 1074).

Dans le programme de 1989, (Education 1989 : 25) l'esthétique incluait plus largement toute image médiatisée : des œuvres d'art, des affiches, des magazines, des livres de contes, des publicités, des bandes dessinées, des vidéos, des images de synthèse, le cinéma et la télévision. L'idée d'une esthétique des beaux-arts européens fondée sur des modèles classiques avait disparu.

L'esthétique revint à l'ordre du jour dans le programme de l'année 2000 (Board of Studies 2000). L'objectif basé sur les modèles esthétiques d'éducation artistique était d'aligner les pratiques artistiques scolaires sur les pratiques artistiques disciplinaires. L'éducation artistique s'est orientée vers la communauté artistique pour trouver des exemples de pratiques et les disciplines académiques comme la critique d'art, l'histoire et la sociologie ont influencé les idéaux de l'éducation artistique. Complétant cette tendance, des programmes spéciaux d'artistes à demeure furent introduits dans les écoles afin de favoriser l'intérêt de la collectivité pour la recherche esthétique et pour fournir des modèles professionnels aux enfants.

\section{L'art porteur de symboles}

L'introduction de la télévision et l'intérêt croissant pour la communication mondiale a porté l'attention vers les aspects communicatifs et symboliques de l'art. Les nouvelles technologies ont promu de nouveaux codes de communication. L'art fut alors considéré dans l'enseignement comme un lieu de rencontre pour de multiples codes de communication, dont les codes figuratifs, iconographiques, narratifs et de composition.

La prise de conscience du pouvoir symbolique de l'art s'est manifestée pour la première fois dans les programmes scolaires australiens en 1974 (Wales 1974). Le texte propose une section sur le langage visuel et, alors que

8. K-6: kindergarten to 6 th grade : de la maternelle à la $6{ }^{\mathrm{e}}$. 
l'intérêt porte sur les fondamentaux théoriques (Wales 1974 : 23), il reconnaît que «l'artiste exprime ses pensées et ses sentiments en termes de langage visuel».

\section{L'art en tant que médiateur culturel}

L'idée que l'art est un médiateur culturel prend forme avec l'opinion selon laquelle tradition et «sens» esthétiques peuvent être nourris par l'art. Selon ce point de vue, l'enseignant est un médiateur de la culture qui propose aux enfants des modèles de bon goût acceptés par la société et une sensibilité à la beauté, l'idée étant que le raffinement culturel et le discernement peuvent s'enseigner. Cette croyance est fondée sur l'idée que le développement du goût et de la conscience de l'existence d'un héritage culturel sont des aspects essentiels pour comprendre le vécu humain et forger une identité culturelle. Tout en croyant fermement que l'éducation artistique développe la prise de conscience culturelle, il y avait un phénomène de reconnaissance de l'art comme puissance civilisatrice. L'objectif culturel de l'éducation artistique reflète l'importance de l'art en tant que legs de civilisations passées et patrimoine national. De cette manière, l'art se hisse au rang de force politique dès lors qu'il est utilisé pour éveiller le sentiment national, et il devient un puissant instrument de propagande. L'éducation artistique en Australie a ainsi transmis une identité et une culture nationales à travers le chant populaire traditionnel, l'étude de peintures australiennes «classiques» et l'apprentissage de la danse traditionnelle australienne (bush dancing).

S'ajoute à cette approche l'idée d'une démocratisation de la culture pouvant s'accomplir grâce à l'éducation artistique. Traditionnellement, l'éducation artistique était un signe de culture souvent réservé aux classes moyennes et supérieures. Dans un programme démocratique, tous les enfants, sans exception, recevaient une éducation culturelle, plus particulièrement en relation avec le développement d'une appréciation de l'art, car on croyait garantir ainsi une meilleure équité au sein de la société. Le programme des arts créatifs (du CP au CE 2) de l'année $2000^{9}$ (Board of Studies 2000) favorise une perspective culturelle et d'une identité nationale fortes.

La logique et les objectifs des programmes scolaires australiens actuels sont dominés par l'opinion que l'art exerce une action sociale.

L'art a également fait son apparition en Australie dans la culture des enfants du baby boom qui a suivi la Seconde Guerre mondiale, comme un moyen d'expression pour la jeunesse et pour une culture alternative. Les jeunes voyaient dans l'art un moyen de se forger une identité. 
Ce changement apparaît dans le programme de 1989 (Education 1989: 25) qui indique que les enfants devraient étudier des modèles, éléments constitutifs du programme d'éducation artistique : des affiches, des publicités, des bandes dessinées, des vidéos, des images de synthèse, des films et des programmes de télévision. De telles suggestions étaient fondées sur l'idée que les modèles artistiques traditionnels n'intéressaient plus les enfants ou ne reflétaient plus leurs valeurs culturelles. Il est intéressant de noter que le programme actuel (Board of Studies 2000) s'est de nouveau tourné, dans son contenu et sa mise en œuvre, vers les modèles traditionnels. Par exemple, pour la classe de CE 1 (Board of Studies 2000 : 57), il est fait référence à l'importance d'étudier les «traditions paysagères, les traditions figuratives ainsi que d'autres traditions et techniques.»

\section{Le postmodernisme}

Il n'est pas possible de donner une définition au postmodernisme au sein de l'éducation artistique en Australie car il reflète un renouveau critique des traditions artistiques dans un sens plus large. Le postmodernisme implique le rôle central de la culture dans toute interprétation des arts, considérant que les analyses critiques et culturelles sont plus significatives que les analyses formelles.

Les idées provenant de différents individus et de différentes cultures peuvent être adaptées à une société toujours plus diversifiée. Plus particulièrement, le postmodernisme remet en question l'autorité des discours d'autrefois sur l'éducation artistique. Les productions et les procédés artistiques (et par conséquent leur enseignement) deviennent des espaces incertains, vagues, confus et interdépendants. Les techniques et la technologie perdent de leur importance au profit des intentions et significations.

Alors que le programme australien lutte encore contre les effets du postmodernisme sur l'éducation artistique, le postmodernisme reflète la culture de la jeunesse et s'y reflète à la fois. La culture de la jeunesse australienne possède ses propres formes artistiques et elle dérange les analyses et critiques dominantes des adultes. Il s'agit d'une explosion artistique, résultant d'un «coup de canon» de la jeunesse tout entière, qui doit inévitablement changer le regard porté par les professeurs australiens sur l'éducation artistique.

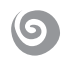

Le défi de l'éducation artistique réside dans sa capacité d'adaptation pour affronter la question du changement social et adopter une perspective critique sur ce qui fait sens dans la culture. 
Il est possible de mettre en place un apprentissage de qualité des disciplines artistiques et de la culture en Australie mais, très souvent, les critères d'évaluation utilisés sont loin de garantir un niveau de qualité acceptable.

En tant que force sociale, l'art est un facteur de changement dans l'enseignement australien : il donne la possibilité aux enfants de se construire une position sociale et de gérer les complexités de notre société moderne. L'art s'inscrit dans un contexte social plus large, il s'intègre à d'autres formes de création artistique comme la littérature, la musique, le théâtre pour forger l'identité des individus. Les approches transdisciplinaires regroupent des disciplines relatives à l'identité culturelle.

La qualité de l'éducation artistique contribue à préparer les étudiants australiens aux exigences toujours changeantes de la société du savoir à venir, et construit les capacités innovatrices et critiques nécessaires pour participer entièrement aux industries culturelles et créatives. Ces principes reconnaissent la valeur de l'éducation artistique en admettant son rôle essentiel, qui est d'offrir l'opportunité de développer son potentiel créatif; de déterminer et soutenir l'avenir artistique et culturel de l'Australie; de construire des compétences critiques, culturelles et symboliques qui favorisent la perception et l'acceptation de soi, d'autrui, et de l'environnement; d'offrir les connaissances essentielles à une société du savoir, de l'avenir et de ses innovations, des industries de la création et de la culture.

\section{BibLIOgRAPHIE}

ASHTON L. (1998): I can't draw to save myself. Australian Institute of Art Education, University of Wollongong.

ASHTON L. (1999): "Deconstructing the aesthetic discourse of drawing: A study of generalist primary teachers in transition.” Australian Art Education, 22 (2) : 41-61.

BAMFORD A. (2006): The Wow Factor: Global research compendium on the impact of the arts in education. Berlin, Waxmann Verlag.

Board of Studies, N. S. W. (2000) : Creative Arts K-6 Syllabus. Sydney, Board of Studies, N. S. W.

BOUGHTON D. (1998): Australian visual arts education: Long standing tensions between socio-cultural realities and government policy. Curriculum, culture and art education: comparative perspectives. K. Freedman and F. Hernandez. New York, State University of New York Press.

BOUGHTON D. (1999) : "How to build an art teacher (1986)." Australian Art Education, $22(1): 59-67$.

BRADY L., SEGAL G., et al. (1998): "Student perceptions of the theory and practice nexus in teacher education." Educational Practice and Theory Into Practice, 20 (1) : 5-16.

DIAMOND P. C. T. and MULLEN C. A. (1999): The Post-modern Educator: Arts-based inquiries and teacher development. New York, Peter Lang.

DUNCUM P. (1999): "Primary art pedagogy: Everything a generalist teacher needs to know." Australian Art Education, 21 (3) : 15-23. 
Education, N. S. W. D. o. (1989): Visual Arts Syllabus and Support Statements. Sydney, Macquarie Publications Pty Ltd.

EFLAND A. (1990): A history of art education: Intellectual and social currents in teaching the visual arts. New York, Teacher's College Press.

ERRAZURIZ L. (1998): Rationales for art education in Chilean schools. Curriculum, culture and art education: comparative perspectives. K. Freedman and F. Hernandez. New York, State University of New York Press.

FREEDMAN K. (1987): Art education as social production: Culture, society and politics in the formation of curriculum. The formation of school subjects: he struggle for creating an American institution. T. Popkewitz. Philadelphia, The Falmer Press, pp. 63-84.

FREEDMAN K. and HERNANDEZ F., Eds. (1998) : Curriculum, culture and art education: Contemporary perspectives. New York, State University of New York Press.

GREER D. W. (1992): "Harry Broudy and Disciplined-Based Art Education (DBAE)." Journal of Aesthetic Education, 26 (4) : 49-60.

HAMBLEN K. and GALANES C. (1997): "Instructional options for aesthetics: Exploring the possibilities." Art Education, 50 (1) : 75-83.

HILLER P. (1999): "Current concerns in art education: Accountability, rationale, relevance, teacher education, etc. (1984).” Australian Art Education, 22 (1) : 34-40.

HOLT D. (1997): "Problems in primary art education: Some reflections on the need for an approach in the early years." International Journal of Early Years Education, 5 (2) : 93-100.

QUINN P. E. (1905): The art reader. Sydney, E.J. Forbes.

SMITH R. (1995): “The question of modernism and postmodernism." Art Education Policy Review, 96 (6) : 2-11.

SPECK C. (1999): “A depth art curriculum for the primary school (1989)." Australian Art Education, 22 (1) : 71-85.

STANKIEWICZ M. A. (1997): Historical research methods in art education. Research methods and methodologies for art education. S. La Pierre, D. and E. Zimmerman. Reston, Virginia, The National Art Education Association, pp. 57-73.

TAYLOR R. (1986): Educating for critical response and development. London, Longman.

THISTLEWOOD D. (1998): From imperialism to internationalism: Policy making in British art education. Curriculum, culture and art education: comparative perspectives. K. Freedman and F. Hernandez. New York, State University of New York Press.

Wales, B. o. S. N. S. (2000): Creative Arts K-6 Syllabus and Units of Work. Sydney, Board of Studies New South Wales.

Wales, D. o. E. N. S. (1952): Curriculum for Primary Schools. Sydney, A.H. Pettifer, Government Printer.

Wales, D. o. E. N. S. (1974): Visual Arts: Curriculum for primary schools. Sydney, Government Printers.

WRIGHT S. (1989): "We've got it backwards: It's too late by age 8." Australian Art Education, 13 (1) : 6-10. 